\title{
MIP models for the irregular strip packing problem: new symme- try breaking constraints
}

\author{
Marcos Okamura Rodrigues ${ }^{1, \star}$, Luiz Henrique Cherri ${ }^{1, \star \star}$, and Leandro Resende Mundim ${ }^{1, \star \star \star}$ \\ ${ }^{1}$ Instituto de Ciências Matemáticas e de Computação, Universidade de São Paulo, Avenida Trabalhador \\ São-carlense, 400 - Centro, São Carlos, Brazil
}

\begin{abstract}
The irregular strip packing problem consists in minimizing the length used to cut a set of pieces from a board with fixed width. Recently, a mixed integer programming model was proposed for the problem, but it may allow a large number of symmetric solutions. In this paper, new symmetry breaking constraints are proposed to improve the model. Computational experiments were performed for instances with convex pieces. The results show the proposed formulation is better than the previous one for most instances, since it improves lower bounds and reduces run-time and number of nodes explored to prove optimality.
\end{abstract}

\section{Introduction}

The irregular strip packing problem (also known as irregular open dimensional problem) consists in cutting irregular pieces from a rectangular board with fixed width and infinite length. In the problem, pieces cannot overlap and each piece must be inside the board. The objective is to minimize the board length used to cut the pieces. The problem is both economical and environmental relevant, since reducing material consumption decreases production costs and minimizes wastes.

The irregular strip packing problem is NP-hard [9] and the methods proposed to solve it are mainly heuristics. The best solutions for large benchmark instances were found in [4]. He proposed a guided cuckoo search algorithm to solve the problem. In his method, the best fit between two pieces is found using a pairwise clustering and then the guided cuckoo search algorithm is applied to find complete solutions.

Some mixed integer programming (MIP) models were proposed to represent the problem. Fischetti and Luzzi [5] were the first to propose a MIP model to the problem. Alvarez-Valdes et al. [1] defined some structures used in [5] and developed a branch-and-bound algorithm to solve the model. Santoro and Lemos [11] proposed a MIP model to convex polygons with some limitations: the polygons can have up to eight edges and their opposite edges must be parallel. Cherri et al. [3] proposed two models that allow rotation of pieces. The models differ in the geometric structures used to avoid overlap among pieces. Toledo et al. [12] proposed the dotted-board model where the pieces were placed in a finite set of dots inside the board. The optimality of the model is subject to the

\footnotetext{
^e-mail: okamura@icmc.usp.br

$\star \star$ e-mail: lhcherri@icmc.usp.br

$\star \star \star$ e-mail: mundim@icmc.usp.br
} 
discretization used to generate the dots. In order to combine the accuracy of continuous models with the advantages of the dotted-board model, Leao et al. [8] proposed a semi-continuous model where the pieces can be placed in continuous positions in $\mathrm{x}$-axis and in discrete positions in $\mathrm{y}$-axis.

Here, new symmetry breaking constraints for the MIP model described in [3] are proposed. Computational results show the performance of the model is improved in various aspects such as lower bounds, number of nodes explored and run-time.

\section{MIP model}

In this section, we define the irregular strip packing problem and review the No-Fit Polygon Covering Model (NFP-CM) proposed in [3].

Given a set of $N$ pieces and a board with fixed width $W$, the irregular strip packing problem consists in minimizing the length $z$ of the board used to cut all pieces. Each piece is defined by a set of vertices in clockwise orientation and one of the vertices is chosen to be its reference point. Let $w_{i}^{\min }$ $\left(w_{i}^{\max }\right)$ be the vertical distance between the reference point of piece $i$ and the vertex of the piece that is nearest (farthest) to the origin. Likewise, let $\ell_{i}^{\min }\left(\ell_{i}^{\max }\right)$ be the distance of the reference point of piece $i$ and the leftmost (rightmost) vertex of the piece. Consider $\left(x_{i}, y_{i}\right)$ the variables that define the translation of the reference point of piece $i$ to place it in the board. Figure 1 illustrates the distance parameters of a piece in NFP-CM.

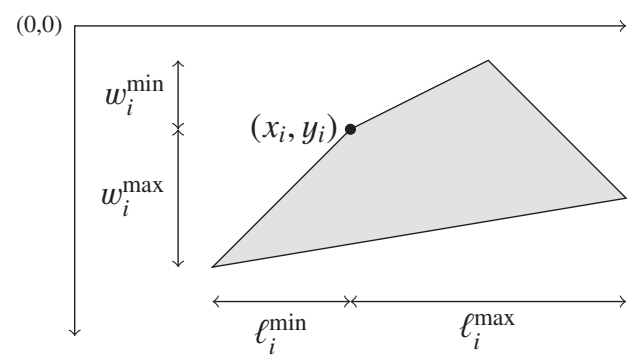

Figure 1: Representation of a piece and its distance parameters in NFP-CM.

The main difficulty to solve the problem is the non-overlap constraints. In NFP-CM the no-fit polygon is used to prevent overlap. For a review about non-overlap techniques and the geometry of the problem, see [2].

[3] defined the following binary variables to prevent overlap:

$$
\xi_{i j}^{k}= \begin{cases}1, & \text { if piece } j \text { is on the right side of edge } k \text { of } N F P_{i j} \\ 0, & \text { otherwise }\end{cases}
$$

where $N F P_{i j}$ is the no-fit polygon between pieces $i$ and $j$. If piece $i$ is on the right side of at least one edge $k=1, \ldots, K_{i j}$ of $N F P_{i j}$, there is no overlap between pieces $i$ and $j$.

NFP-CM is given by:

$\min z$ 
s.t.

$$
\begin{array}{ll}
\ell_{i}^{\min } \leq x_{i} \leq z-\ell_{i}^{\max }, & i=1, \ldots, N, \\
w_{i}^{\min } \leq y_{i} \leq W-w_{i}^{\max }, & i=1, \ldots, N, \\
a_{i j}^{k}\left(x_{i}-x_{j}\right)+b_{i j}^{k}\left(y_{i}-y_{j}\right) \leq c_{i j}^{k}+M_{i j}^{k}\left(1-\xi_{i j}^{k}\right), & 1 \leq i<j \leq N, k=1, \ldots, K_{i j}, \\
\sum_{k=1}^{K_{i j}} \xi_{i j}^{k}=1, & 1 \leq i<j \leq N, \\
\xi_{i j}^{k} \in\{0,1\}, & 1 \leq i<j \leq N, k=1, \ldots, K_{i j} .
\end{array}
$$

Objective function (2) minimizes the length used to cut all pieces. Constraints (3) and (4) impose the pieces are placed inside the board. Constraints (5) prevent overlap among pieces. Constraints (6) ensure each piece is on the right side of an edge of the no-fit polygon while constraints (7) define the domain of binary variables.

\section{Symmetry breaking constraints}

NFP-CM (2)-(7) may have infinite symmetric solutions. Therefore, identical or equivalent solutions can be found several times by the enumeration scheme. In this section symmetric breaking constraints are proposed. The constraints can improve the linear relaxation of the model and reduce the number of identical solutions.

\subsection{Partitioning the solution space}

The main drawback of NFP-CM is non-overlap constraints (5). The definition of variables $\xi_{i j}^{k}$ generates overlap between various regions, as shown in Figure 2(a). Dotted regions are common to two binary variables and hatched regions are common to three variables.

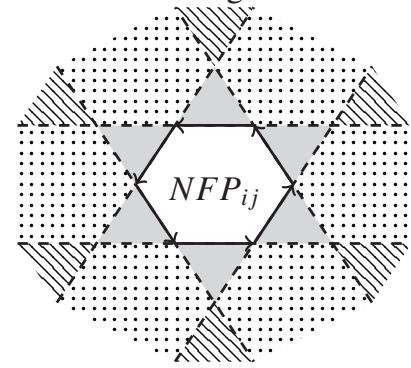

(a)

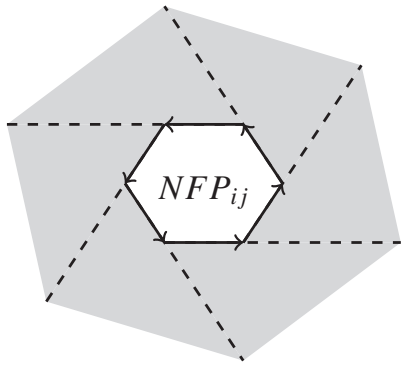

(b)

Figure 2: Non-overlap regions. (a) NFP-CM model. (b) Proposed partitioning.

Such redundancies on $\xi_{i j}^{k}$ values can be avoided by adding constraints (8) to the model. The constraints ensure the reference point of piece $j$ is on the left side of the line defined by edge $k^{\prime}$, where edge $k^{\prime}$ precedes edge $k$ in the no-fit polygon. Therefore, the reference point of piece $j$ can activate only one $\xi_{i j}^{k}$ variable. Figure 2(b) illustrates the partitioning obtained from a no-fit polygon.

$$
\bar{a}_{i j}^{k^{\prime}}\left(x_{i}-x_{j}\right)+\bar{b}_{i j}^{k^{\prime}}\left(y_{i}-y_{j}\right) \leq \bar{c}_{i j}^{k^{\prime}}+\bar{M}_{i j}^{k^{\prime}}\left(1-\xi_{i j}^{k}\right), \quad 1 \leq i<j \leq N, k=1, \ldots, K_{i j},
$$




\subsection{Connecting pieces}

In a feasible solution for the irregular strip packing problem, some pieces may not touch each other. However, one can translate the pieces to connect them, obtaining another solution with the same quality. Consider the following variables:

$$
\phi_{i j}= \begin{cases}1, & \text { if piece } i \text { is connected to piece } j \\ 0, & \text { otherwise }\end{cases}
$$

Constraints (10) combined with (5) ensure if $\phi_{i j}$ is equal to 1 , the pieces $i$ and $j$ are connected.

$$
a_{i j}^{k}\left(x_{i}-x_{j}\right)+b_{i j}^{k}\left(y_{i}-y_{j}\right) \geq c_{i j}^{k}-\underline{M}_{i j}^{k}\left(1-\phi_{i j}\right), \quad 1 \leq i<j \leq N, k=1, \ldots, K_{i j} .
$$

To ensure each piece is connected with another piece, the following constraints are imposed:

$$
\sum_{j=1}^{i-1} \phi_{j i}+\sum_{j=i+1}^{N} \phi_{i j} \geq 1, \quad i=1, \ldots, N
$$

Note that constraints (11) do not ensure all pieces are connected as a single component. For example, it may have two groups of connected pieces with no interconnection. To enforce all pieces are connected, one can impose all cut sets of pieces are connected, as defined by constraints (12).

$$
\sum_{(i, j) \in \delta(S) \mid i<j} \phi_{i j} \geq 1, \quad \forall S \subset N, S \neq \emptyset
$$

where $\delta(S)$ is the cutset of $S$.

Although constraints (12) impose connectivity, they introduce an exponential number of constraints in the model and therefore hamper its resolution.

\subsection{Connecting pieces with the board}

In the optimal solution, at least one piece can touch the bottommost position of the board. In fact, if there is a solution that does not satisfy this statement, one can translate a piece down until it touches the board, obtaining a solution with the same quality.

Let $\omega_{i}$ be a binary variable, defined as follows:

$$
\omega_{i}= \begin{cases}1, & \text { if piece } i \text { is in the bottommost position of the board } \\ 0, & \text { otherwise }\end{cases}
$$
board.

Constraints (13) and (14) ensure at least one piece is placed in the bottommost position in the

$$
\begin{gathered}
y_{i} \leq w_{i}^{\min }+\left(W-w_{i}^{\max }-w_{i}^{\min }\right)\left(1-\omega_{i}\right), \quad i=1, \ldots, N, \\
\sum_{i=1}^{N} \omega_{i}=1 .
\end{gathered}
$$




\subsection{Pieces with same shape}

Symmetric solutions can be found if there are multiple pieces with same type (shape). For example, if there are two pieces with same type, all the solutions can be duplicated in an enumeration scheme. To avoid such problem, the pieces of same type can be ordered. Consider $T_{i}$ the type of piece $i$, to eliminate the symmetry on the placement of pieces of same type, the following constraints are imposed.

$$
y_{i} \leq y_{j}, \quad i=1, \ldots, N, j=\min \left\{k=i+1, . ., N \mid T_{k}=T_{i}\right\} .
$$

Note that constraints (15), except in the case where the pieces are placed in the same $y$-position, eliminate symmetry of identical pieces.

Some values of $\omega_{i}$ can be inferred for pieces with same type using constraints (13)-(15). Specifically, for a piece with several copies, only the smallest index constraint (13) can be active. Constraints (16) ensure only the smallest index copy of each piece type can activate constraints (13).

$$
\omega_{j}=0, \quad i=1, \ldots, N, j=\min \left\{k=i+1, . ., N \mid T_{k}=T_{i}\right\} .
$$

\section{Results}

In this section we present computational experiments to evaluate the performance of the proposed symmetry breaking constraints. The experiments were run on a computer with Intel ${ }^{\circledR}$ Core $^{\mathrm{TM}}$ i7-2600 @ 3.40GHz CPU and 16 GB RAM under Ubuntu 14.04 LTS 64 bits operating system. The instances were solved by IBM ILOG CPLEX 12.6 solver using default parameters and a time limit of 3,600 seconds.

Table 1 presents the main characteristics of the evaluated instances: the name, the author(s) who proposed it, the total number of pieces, the number of piece types, the average number of vertices, and the upper bound (UB). The total number of pieces ranges between 3 and 17, with an average of 8.6 pieces, and an average number of 4.02 vertices per instance. Additionally, we present the upper bound (UB) used to estimate the big-M coefficients in constraints (5), for more details see [3]. UB was calculated using the bottom-left heuristic proposed in [6] with one thousand random input sequences.

Table 2 reports the computational experiments of NFP-CM proposed in [3] and the improved NFP-CM, i.e., NFP-CM with symmetry breaking constraints (8) and (13)-(16). Improved NFP-CM obtained better or equal lower bounds (LB) for all instances. Moreover, improved NFP-CM proved optimality for nineteen instances, four more instances than NFP-CM. In average, improved NFP-CM was $35.70 \%$ faster than NFP-CM to prove optimality. However, improved NFP-CM was slower for instance santoro4. Considering nodes explored by CPLEX, three instances were solved at the root node. For the remaining instances, improved NFP-CM explored fewer nodes for fourteen cases. The biggest reduction of nodes explored to prove optimality was obtained for instance santoro1, where NFP-CM explored 185.36 times the amount of nodes of improved NFP-CM. Furthermore, improved NFP-CM solved all instances santoro faster than the original paper [11]. We also highlight improved NFP-CM is the first MIP model to prove optimality for instances threep3, threep3w9 and fu in less than 1,200 seconds.

\section{Conclusions and further work}

In this paper we have proposed symmetry breaking constraints for the irregular strip packing problem. Although this new set of constraints was added to NFP-CM proposed in [3], the ideas presented here can be applied to other models. 
Table 1: Features of benchmark instances.

\begin{tabular}{llrrrr}
\hline Instance & Author(s) & Pieces & Piece types & Avg. vertices & UB \\
\hline jones1 & Jones [7] & 4 & 4 & 3.25 & 5.00 \\
jones2 & Jones [7] & 6 & 6 & 3.83 & 10.00 \\
three & Alvarez-Valdes et al. [1] & 3 & 3 & 3.67 & 6.67 \\
threep2 & Alvarez-Valdes et al. [1] & 6 & 3 & 3.67 & 9.67 \\
threep2w9 & Alvarez-Valdes et al. [1] & 6 & 3 & 3.67 & 8.67 \\
threep3 & Alvarez-Valdes et al. [1] & 9 & 3 & 3.67 & 14.00 \\
threep3w9 & Alvarez-Valdes et al. [1] & 9 & 3 & 3.67 & 12.00 \\
fu5 & Alvarez-Valdes et al. [1] & 5 & 4 & 3.20 & 37.00 \\
fu6 & Alvarez-Valdes et al. [1] & 6 & 5 & 3.33 & 17.89 \\
fu7 & Alvarez-Valdes et al. [1] & 7 & 6 & 3.43 & 24.00 \\
fu8 & Alvarez-Valdes et al. [1] & 8 & 7 & 3.50 & 24.00 \\
fu9 & Alvarez-Valdes et al. [1] & 9 & 8 & 3.44 & 24.00 \\
fu10 & Alvarez-Valdes et al. [1] & 10 & 9 & 3.50 & 28.00 \\
fu & Alvarez-Valdes et al. [1] & 12 & 11 & 3.42 & 30.00 \\
rco1 & Ribeiro et al. [10] & 7 & 7 & 5.14 & 8.00 \\
rco2 & Ribeiro et al. [10] & 14 & 7 & 5.14 & 15.00 \\
santoro1 & Santoro and Lemos [11] & 11 & 11 & 8.00 & 28.00 \\
santoro2 & Santoro and Lemos [11] & 11 & 10 & 4.55 & 6.20 \\
santoro3 & Santoro and Lemos [11] & 12 & 8 & 4.33 & 6.50 \\
santoro4 & Santoro and Lemos [11] & 17 & 11 & 4.00 & 19.00 \\
\hline & & & & &
\end{tabular}

Table 2: Comparison between MIP models.

\begin{tabular}{|c|c|c|c|c|c|c|c|c|}
\hline \multirow[t]{2}{*}{ Instance } & \multicolumn{4}{|c|}{ NFP-CM } & \multicolumn{4}{|c|}{ Improved NFP-CM } \\
\hline & LB & UB & Time (s) & Nodes & LB & UB & Time (s) & Nodes \\
\hline jones 1 & 5.00 & 5.00 & 0 & 0 & 5.00 & 5.00 & $\mathbf{0}$ & $\mathbf{0}$ \\
\hline jones2 & 10.00 & 10.00 & $\mathbf{0}$ & $\mathbf{0}$ & 10.00 & 10.00 & $\mathbf{0}$ & $\mathbf{0}$ \\
\hline three & 6.00 & 6.00 & $\mathbf{0}$ & 0 & 6.00 & 6.00 & $\mathbf{0}$ & $\mathbf{0}$ \\
\hline threep2 & 9.33 & 9.33 & 2 & 21,360 & 9.33 & 9.33 & 1 & 12,963 \\
\hline threep2w9 & 8.00 & 8.00 & 6 & 107,113 & 8.00 & 8.00 & 1 & 15,217 \\
\hline threep3 & 10.06 & 13.53 & 3,600 & $24,408,906$ & 13.53 & 13.53 & 928 & $\mathbf{9 , 7 1 1 , 3 5 8}$ \\
\hline threep3w9 & 8.75 & 11.00 & 3,600 & $33,442,609$ & 11.00 & 11.00 & 1,113 & $9,036,051$ \\
\hline fu5 & 17.89 & 17.89 & 0 & 336 & 17.89 & 17.89 & $\mathbf{0}$ & 235 \\
\hline fu6 & 23.00 & 23.00 & $\mathbf{0}$ & 428 & 23.00 & 23.00 & $\mathbf{0}$ & 452 \\
\hline fu7 & 24.00 & 24.00 & $\mathbf{0}$ & 1,324 & 24.00 & 24.00 & 0 & 1,481 \\
\hline fu8 & 24.00 & 24.00 & 0 & 851 & 24.00 & 24.00 & 0 & 3,127 \\
\hline fu9 & 25.00 & 25.00 & 7 & 83,709 & 25.00 & 25.00 & 4 & 32,911 \\
\hline fu10 & 28.69 & 28.69 & 301 & $2,653,576$ & 28.69 & 28.69 & 33 & 253,314 \\
\hline fu & 28.00 & 33.14 & 3,600 & $18,761,708$ & 33.14 & 33.14 & 511 & $2,539,903$ \\
\hline rcol & 8.00 & 8.00 & 1 & 14,127 & 8.00 & 8.00 & 1 & 12,008 \\
\hline $\mathrm{rco} 2$ & 6.67 & - & 3,600 & $17,116,136$ & 9.00 & - & 3,600 & $10,534,103$ \\
\hline santoro1 & 26.85 & 28.00 & 3,600 & $49,058,509$ & 28.00 & 28.00 & 41 & 264,666 \\
\hline santoro2 & 6.00 & 6.00 & 474 & $6,958,452$ & 6.00 & 6.00 & 19 & 120,888 \\
\hline santoro3 & 6.00 & 6.00 & 5 & 30,938 & 6.00 & 6.00 & 5 & 17,955 \\
\hline santoro4 & 15.00 & 15.00 & 24 & 178,258 & 15.00 & 15.00 & 52 & 131,688 \\
\hline Optimal & & & 15 & & & & 19 & \\
\hline
\end{tabular}


Computational experiments show the relevance of this new set of constraints. In general, the symmetry breaking constraints improve lower bounds and reduce run-time and the number of nodes explored to prove optimality.

For further work, we intend to introduce symmetry breaking constraints for instances with nonconvex pieces and study branching priorities for binary variables.

\section{Acknowledgements}

This research was supported by São Paulo Research Foundation (FAPESP), grants \#2015/24987-4, \#2014/23900-0, \#2013/07375-0 \#2012/18653-8 and \#2010/10133-0 and Brazilian Federal Agency for Support and Evaluation of Graduate Education (CAPES).

\section{References}

[1] R. Alvarez-Valdes, A. Martinez, and J.M. Tamarit. A branch \& bound algorithm for cutting and packing irregularly shaped pieces. International Journal of Production Economics, 145(2):463 $-477,2013$.

[2] Julia A. Bennell and Jose F. Oliveira. The geometry of nesting problems: A tutorial. European Journal of Operational Research, 184(2):397 - 415, 2008.

[3] Luiz Henrique Cherri, Leandro Resende Mundim, Marina Andretta, Franklina M. B. Toledo, José F. Oliveira, and Maria Antonia Carravilla. Mixed-integer programming models for nesting problems. European Journal of Operational Research, 253(3):570-583, 2016.

[4] Ahmed Elkeran. A new approach for sheet nesting problem using guided cuckoo search and pairwise clustering. European Journal of Operational Research, 231(3):757 - 769, 2013.

[5] Matteo Fischetti and Ivan Luzzi. Mixed-integer programming models for nesting problems. Journal of Heuristics, 15(3):201-226, 2009.

[6] A. M. Gomes and J. F. Oliveira. Solving irregular strip packing problems by hybridising simulated annealing and linear programming. European Journal of Operational Research, 171(3): $811-829,2006$.

[7] Donald R. Jones. A fully general, exact algorithm for nesting irregular shapes. Journal of Global Optimization, 59(2):367-404, 2014.

[8] Aline A.S. Leao, Franklina M.B. Toledo, José Fernando Oliveira, and Maria Antónia Carravilla. A semi-continuous mip model for the irregular strip packing problem. International Journal of Production Research, 54(3):712-721, 2016.

[9] B. K. Nielsen and A. Odgaard. Fast neighbourhood search for nesting problem. Technical Report 03/02, DIKU, Department of Computer Science, University of Copenhagen, 2003.

[10] Cristina Ribeiro, Maria Antónia Carravilla, and José F. Oliveira. Applying constraint logic programming to the resolution of nesting problems. Pesquisa Operacional, 19(2):239-247, 1999.

[11] M. C. Santoro and F. K. Lemos. Irregular packing: Milp model based on a polygonal enclosure. Annals of Operations Research, 235(1):693-707, 2015.

[12] F. M. B. Toledo, M. A. Carravilla, C. Ribeiro, J. F. Oliveira, and A. M. Gomes. The dottedboard model: a new mip model for nesting irregular shapes. International Journal of Production Economics, 145(2):478 - 487, 2013. 